\title{
ANÁLISE DA EFICIÊNCIA TÉCNICA NAS LAVOURAS DE ARROZ NO RIO GRANDE DO SUL
}

\section{ANALYZING THE TECHNICAL EFFICIENCY OF RICE FARMS IN THE RIO GRANDE DO SUL}

\author{
Augusto Mussi Alvim \\ Pontifícia Universidade Católica do Rio Grande do Sul - Porto Alegre - RS - Brasil \\ Valter José Stülp \\ Pontifícia Universidade Católica do Rio Grande do Sul - Porto Alegre - RS - Brasil \\ Victor Hugo Kayser \\ Instituto Riograndense do Arroz - General Camara - RS - Brasil
}

Resumo: O presente artigo avalia a eficiência técnica nas lavouras de arroz no Rio Grande do Sul-RS. Para isso, utilizou-se a Análise Envoltória de Dados (Dalta Envelopment Analysis)-DEA e o modelo de regressão Tobit. $O$ estudo utilizou as variáveis disponíveis no Censo da Lavoura de Arroz Irrigado do RS (IRGA, 2006). Os resultados da pesquisa mostram que a maior parte das lavouras de arroz é ineficiente tecnicamente em termos locais, apenas $5,7 \%$ são eficientes. Além disso, observa-se que as principais variáveis capazes de aumentar o nível de eficiência nas diferentes regiões do estado são: assistência técnica, nível de escolaridade e sistema de cultivo. Os resultados mostram que existem diferenças importantes entre as lavouras de arroz conforme a região do estado e a condição do produtor (proprietário ou arrendatário). Palavras-chave: Eficiência técnica. DEA. Tobit e lavoura de arroz.

Abstract: This paper evaluates the technical efficiency in rice farms in Rio Grande do Sul (RS). For this, we use the Data Envelopment Analysis (DEA) and the Tobit regression model. The study use the variables available in the Census of the Institute of Irrigated Rice of RS (IRGA, 2006). The study shows that most of the rice farmers are technically inefficient on local terms, and only $5.7 \%$ are efficient. In addition, it is possible to observe that the main variables, which increase the level of efficiency in different regions of the state, are technical assistance, education level and growing system. The results show that there are important differences between the rice farms, which depends of the region of RS and of the producer condition (owner or tenant).

Keywords: Technical efficiency. DEA. Tobit and rice farming.

\section{INTRODUÇÃO}

Redes (St. Cruz Sul, Online), v. 20, n² 2, p. 158 - 175, maio/ago. 2015158 
Apesar de atender a uma parcela significativa da demanda brasileira por arroz, a atividade orizícola no Rio Grande do Sul-RS parece ser ineficiente e inadequada frente aos seus parceiros comerciais os produtores da Argentina e do Uruguai. Associado a isso, há o interesse por parte dos produtores gaúchos de ampliar o seu mercado, incrementando as exportações e reduzindo os riscos da atividade. Dada à escassez de recursos naturais (terra e água) existe uma preocupação crescente da sociedade em avaliar o desempenho das atividades agrícolas, buscando um melhor desempenho econômico, social e ambiental.

Um caminho para avaliar a produção de arroz no RS pode ser feito a partir da eficiência técnica e da identificação das principais variáveis na atividade que permitem incrementar o nível de eficiência. Além de apurar a eficiência relativa das lavouras de arroz no RS, o presente estudo pretende identificar as variáveis relevantes que têm potencial para incrementar a eficiência técnica.

Nesse sentido, o presente estudo analisa a eficiência técnica no uso dos recursos disponíveis nas lavouras de arroz do RS, comparando para as diversas lavouras, como os fatores de produção são transformados em produtos. Para atingir tal objetivo é utilizada a DEA, baseada em programação linear. Essa técnica tem se revelado uma alternativa relevante para estudar a eficiência técnica em ambientes complexos, como é o caso da atividade orizícola no RS. É utilizado, também, o modelo Tobit a fim de identificar as variáveis mais relevantes e capazes de melhorar os níveis de eficiência nessa atividade.

A seguir, a seção 2 apresenta uma revisão bibliográfica, analisando os principais estudos que aplicam essa metodologia, as variáveis incluídas no modelo e os principais resultados. Na seção 3 do trabalho, é detalhado o modelo DEA e Tobit. Nessa seção, também são definidas as fontes dos dados utilizados, conceitos e testes realizados. $\mathrm{Na}$ seção 4, são discutidos os principais resultados obtidos, com destaque ao nível de eficiência e as variáveis que explicam as diferenças entre as regiões do RS. Por fim, são feitas as considerações finais.

\section{REVISÃO BIBLIOGRÁFICA}


Nesta seção, são apresentados os principais estudos que avaliam questões relativas à atividade agropecuária e à eficiência técnica. Destaca-se as diferentes metodologias utilizadas, a escolha das variáveis e os resultados obtidos em estudos recentes sobre esta temática.

Arnade (1998) analisa a evolução da eficiência técnica e da produtividade total dos fatores de produção do setor agrícola para 70 países no período de 1961 a 1993. A eficiência técnica é estimada por meio do método DEA. Os resultados mostram que, nos países desenvolvidos, a agricultura é eficiente e a produtividade dos fatores tem aumentado. Entretanto, em muitos países em desenvolvimento, a agricultura permanece ineficiente e a produtividade total dos fatores tem diminuído. Nesse grupo de países, o produto agrícola tem aumentado devido ao maior uso de máquinas e fertilizantes.

Coelli, Rahman e Thirtle (2002) analisam a eficiência técnica, de escala, alocativa e econômica para 406 propriedades produtoras de arroz de Bangladesh, através do modelo DEA. Por meio de regressão com o uso do modelo Tobit, os autores procuram identificar as variáveis que afetam os índices de eficiência das propriedades rurais. As propriedades com famílias grandes possuem maior probabilidade de serem ineficientes, indicando a dificuldade para encontrar emprego fora da propriedade. Os proprietários que têm maior acesso aos mercados de insumos e os que exercem menos tarefas fora da propriedade tendem a ser mais eficientes. As variáveis como idade, educação, experiência, fertilidade do solo, serviços de extensão e treinamento não apresentam influência significativa sobre os índices de eficiência.

Já Ferreira Filho e Pereira Filho (2003) calculam a eficiência técnica, de escala, alocativa e econômica para 44 produtores agrícolas do Recôncavo da Bahia. Os autores concluem que $84 \%$ dos produtores são tecnicamente eficientes, $80 \%$ eficientes de escala e somente $25 \%$ são eficientes em termos alocativos. Dessa forma, os autores verificam que a ineficiência econômica é devido à ineficiência alocativa $(78,4 \%)$, de escala $(12,6 \%)$ e técnica (9\%). Os produtores analisados são, na grande maioria, tecnicamente eficientes, estando próximos ou na fronteira de produção da tecnologia atual. Assim sendo, a maneira de aumentar a produtividade marginal por meios técnicos é possível por meio da mudança tecnológica. 
Dhungana, Nuthall e Nartea (2004) analisam a eficiência técnica, de escala, alocativa e econômica de 76 produtores de arroz do Nepal, utilizando o modelo DEA. Numa segunda etapa, com um modelo de regressão Tobit, avaliam a influência da idade, nível de educação, grau de aversão ao risco, quantidade de mão de obra da família e sexo do dirigente da propriedade sobre o índice de eficiência. Quanto ao fator idade, concluem que a probabilidade de os jovens serem ineficientes é maior do que a dos mais velhos, talvez devido à maior experiência adquirida pelos últimos. Os produtores com maior nível educacional são os mais eficientes.

Os autores concluem que quanto maior o grau de aversão ao risco do produtor, maior é a probabilidade de ser ineficiente em termos econômicos, alocativos e em escala, e maior a probabilidade de ser eficiente tecnicamente. Os produtores com maior aversão ao risco tendem a utilizar mais os insumos da propriedade (terra, sementes e mão de obra) e menos os insumos comprados (fertilizantes, máquinas, pesticidas), sendo mais eficientes tecnicamente. A relação entre o percentual da mão de obra familiar no total empregado na propriedade e a eficiência é positiva, apesar de não ser significante estatisticamente. Isso talvez ocorra porque parte da mão de obra familiar ajuda a suprir as necessidades desse fator em épocas de grande demanda. As mulheres, como dirigentes da propriedade, mostram-se menos ineficientes do que os homens em termos econômicos, alocativos e de escala.

Vicente (2004) analisa a eficiência técnica, alocativa e econômica dos estados brasileiros na produção agrícola, com dados do Censo Agropecuário de 1995/96. A estimativa de eficiência é realizada por meio do modelo DEA. Os estados do Sudeste, Centro-Oeste e Sul apresentam índices de eficiência acima da média nacional, enquanto os índices dos estados do Nordeste e Norte são os mais baixos. Por meio de um modelo de regressão Tobit, o autor identifica as variáveis que explicam os diferentes níveis de eficiência. A eficiência técnica é explicada por variáveis associadas às características do solo, às condições climáticas e à irrigação. Já o nível de educação influencia a eficiência alocativa. A eficiência econômica é mais influenciada pela eficiência alocativa do que pela eficiência técnica. A simulação da minimização de custos (eficiência econômica ou eficiência total) mostra 
que a terra e a mão de obra são utilizadas em excesso e que os fertilizantes e os pesticidas são subutilizados.

Helfand e Levine (2004) analisam a eficiência técnica dos estabelecimentos rurais da região Centro-Oeste do Brasil com base nos dados do Censo Agropecuário de 1995/1996. Em uma primeira etapa, a eficiência é estimada por meio do modelo DEA. O produto considerado (output) é o valor da produção agrícola e os insumos (inputs) são: a área de terra, a mão de obra, os tratores, o rebanho e os insumos comprados. Na segunda etapa da análise, os autores procuram explicar a eficiência técnica dos estabelecimentos rurais por meio de regressões em que a variável explicada é o índice de ineficiência.

Os autores observam que existe uma relação em forma de U entre eficiência e tamanho do estabelecimento. Os estabelecimentos com até 50 hectares são os mais eficientes. Estabelecimentos com tamanho entre 200 a 500 hectares são $20 \%$ menos eficientes do que os primeiros. A eficiência diminui com o tamanho do estabelecimento até 2000 há. Após esse tamanho, a eficiência começa a aumentar. A elevação da eficiência nos grandes estabelecimentos deve-se ao acesso facilitado à assistência técnica, ao mercado e à eletrificação rural.

Helfand e Levine (2004) também avaliam o impacto do sistema de posse da terra explorada, embora o efeito desta variável seja pequeno. Os arrendatários da terra que pagam valor pré-fixado pelo arrendamento são $14 \%$ mais eficientes do que os proprietários. Os arrendatários que pagam o arrendamento da terra como um percentual da colheita e os ocupantes da terra são, respectivamente, $5 \%$ e $7 \%$ menos eficientes que os proprietários.

Helfand e Levine (2004) afirmam que a composição do produto também influencia a eficiência do estabelecimento, sendo mais eficientes os que exploram produtos de valor mais elevado como horticultura, suínos, aves, lavouras permanentes e temporárias. Também são mais eficientes os estabelecimentos com acesso ao crédito, ao mercado, à eletricidade, à assistência técnica e às cooperativas. Finalmente, as práticas de mecanização, irrigação, fertilização, conservação do solo, controle de doenças também contribuem para aumentar a eficiência. 
Chavas, Petrie e Roth (2005) analisam a eficiência técnica, alocativa e de escala de um grupo de produtores rurais do Gâmbia. Utilizando um modelo Tobit, os autores constatam que grande parte da ineficiência alocativa é devido às imperfeições existentes nos mercados do capital e da mão de obra. Devido às dificuldades para conseguir crédito, o produtor procura realizar tarefas fora da propriedade para suprir as necessidades de capital. As tarefas na propriedade são alocadas às mulheres que enfrentariam restrições institucionais para assumir certas funções.

Gonçalves, Vieira, Lima e Gomes (2008) analisam a eficiência técnica e de escala de 771 produtores de leite do Estado de Minas Gerais, por meio do método DEA. Utilizam o método de regressão Tobit para explicar os índices de eficiência por meio de um conjunto de variáveis. A maioria dos produtores incluídos na análise apresenta índices de ineficiência. As variáveis que melhoram o índice de eficiência dos produtores que produzem até 50 litros diários de leite são internas à propriedade, tais como: experiência acumulada (expressa pela idade), a produtividade da mão de obra, da vaca e do capital operacional.

Os autores observam que os grandes produtores (com mais de 200 litros diários) são mais eficientes do que os pequenos. As variáveis que aumentam a eficiência daqueles são o acesso ao crédito, assistência técnica e treinamento. Esses fatores não influenciam a eficiência dos pequenos produtores devido ao pouco acesso que eles têm aos mesmos. A diferença de eficiência entre pequenos e grandes produtores é devido, também, ao fato destes últimos terem melhores condições para operar o produto, adicionando qualidade ao mesmo. Maior volume de produto e melhor qualidade significam maior preço.

Em relação à escala de produção, Chavas, Chambers e Pope (2010) comentam que a mecanização agrícola contribui para o aumento no tamanho dos estabelecimentos rurais. Isso levanta a questão da existência ou não de rendimentos de escala e de ganhos de eficiência por parte dos grandes estabelecimentos. A evidência empírica indica que a curva de custo médio dos estabelecimentos rurais tem a forma de L. Pequenos estabelecimentos rurais têm curva de custo médio declinante com o aumento da produção, mas nos estabelecimentos médios e grandes o custo médio se mantém constante. Assim, as 
economias de escala não são um incentivo para o estabelecimento rural de tamanho médio ou grande se tornar ainda maior.

Chavas (2008) afirma que os estabelecimentos rurais menores tendem a compensar os custos médios de produção mais elevados por meio da diversificação da produção. Essa diversificação também reduz o risco. Assim, apesar de haver rendimentos crescentes de escala quando o pequeno estabelecimento aumenta a área, não é possível definir uma dimensão mínima desejável.

Portanto, os diversos estudos vistos acima mostram situações de ineficiência técnica, de escala, alocativa e total (econômica) na agricultura de diversas regiões do mundo. A partir disso, é possível identificar os principais modelos e variáveis utilizados para analisar a eficiência técnica. Na próxima seção, é apresentada a metodologia utilizada no presente trabalho, que se baseou nos estudos apresentados nesta seção para a escolha dos modelos, variáveis e técnicas de análise.

\section{METODOLOGIA DE ANÁLISE}

Os métodos de análise utilizados neste estudo são divididos em duas partes: a primeira parte consiste na estimativa da eficiência técnica na produção de arroz do Rio Grande do Sul por meio do método denominado DEA. A segunda parte procura determinar as variáveis explicativas da eficiência técnica das lavouras orizícolas, por meio de um modelo de regressão Tobit. Para ambas as etapas, os dados utilizados na análise são do Censo da Lavoura de Arroz Irrigado do Rio Grande do Sul- Safra 2004/05 (IRGA, 2006). Este censo foi realizado pelo Instituto Rio Grandense do Arroz, abrangendo 9032 lavouras de arroz, distribuídas em 133 municípios do RS.

\subsection{Análise Envoltória de Dados}

A DEA mede a eficiência de cada Unidade de Tomada de DecisãoUTD na geração do produto (ou produtos) a partir de um conjunto de fatores de produção. Essa eficiência é medida em função da distância de cada UTD em relação à situação ótima na fronteira de eficiência. A UTD pode ser uma firma, uma escola, uma universidade, um hospital, uma 
agência governamental ou uma região geográfica. Neste estudo, a UTD é a lavoura de arroz irrigado do Rio Grande do Sul.

A eficiência pode ser dividida em eficiência técnica e eficiência alocativa. A eficiência técnica consistiria na UTD obter o máximo de produto(s) a partir de um conjunto de fatores de produção, ou então, produzir uma dada quantidade de produto(s) com a quantidade mínima do conjunto de fatores. Essa eficiência técnica pode ser considerada como eficiência técnica local e/ou eficiência técnica global (ver figura 1). $\mathrm{Na}$ eficiência técnica local, considera-se a distância da UTD da fronteira de eficiência, desconsiderando os retornos de escala. Mas, embora a UTD esteja na fronteira de eficiência técnica local, poderia existir a possibilidade de ela aumentar o produto por unidade de fator variando a escala de produção, na situação em houvesse retornos crescentes ou decrescentes de escala. Na eficiência técnica global, a UTD estaria operando na escala ótima de produção.

A eficiência alocativa considera, em vez de um máximo de produto, um máximo de lucro ou receita e, em vez de uma quantidade mínima de fatores de produção, um custo mínimo. Portanto, na eficiência alocativa, os fatores são alocados e as relações entre as quantidades de produtos produzidos variam de acordo com seus preços.

Neste estudo, avalia-se a eficiência técnica das lavouras de arroz sob o ponto de vista da eficiência técnica local, global e de escala. $O$ software utilizado neste estudo é o DEA-SOLVER e os modelos de análise são designados como CCR e BCC, apresentados e descritos por Cooper, Seiford e Tone (2006). ${ }^{1}$

O modelo CCR considera retornos constantes de escala determinando a eficiência técnica global. O modelo BCC considera retornos variáveis de escala determinando a eficiência técnica local da UTD.

Para uma maior compreensão desses tipos de eficiência, segue uma descrição dos mesmos, considerando-se a situação de um produto e um fator de produção. Essa descrição e a figura 1 abaixo são baseados em Cooper, Seiford e Tone (2006).

\footnotetext{
1 O modelo CCR foi proposto inicialmente por Charnes, Cooper e Rhodes (1978).
} 
Figura 1. Eficiência técnica global e local

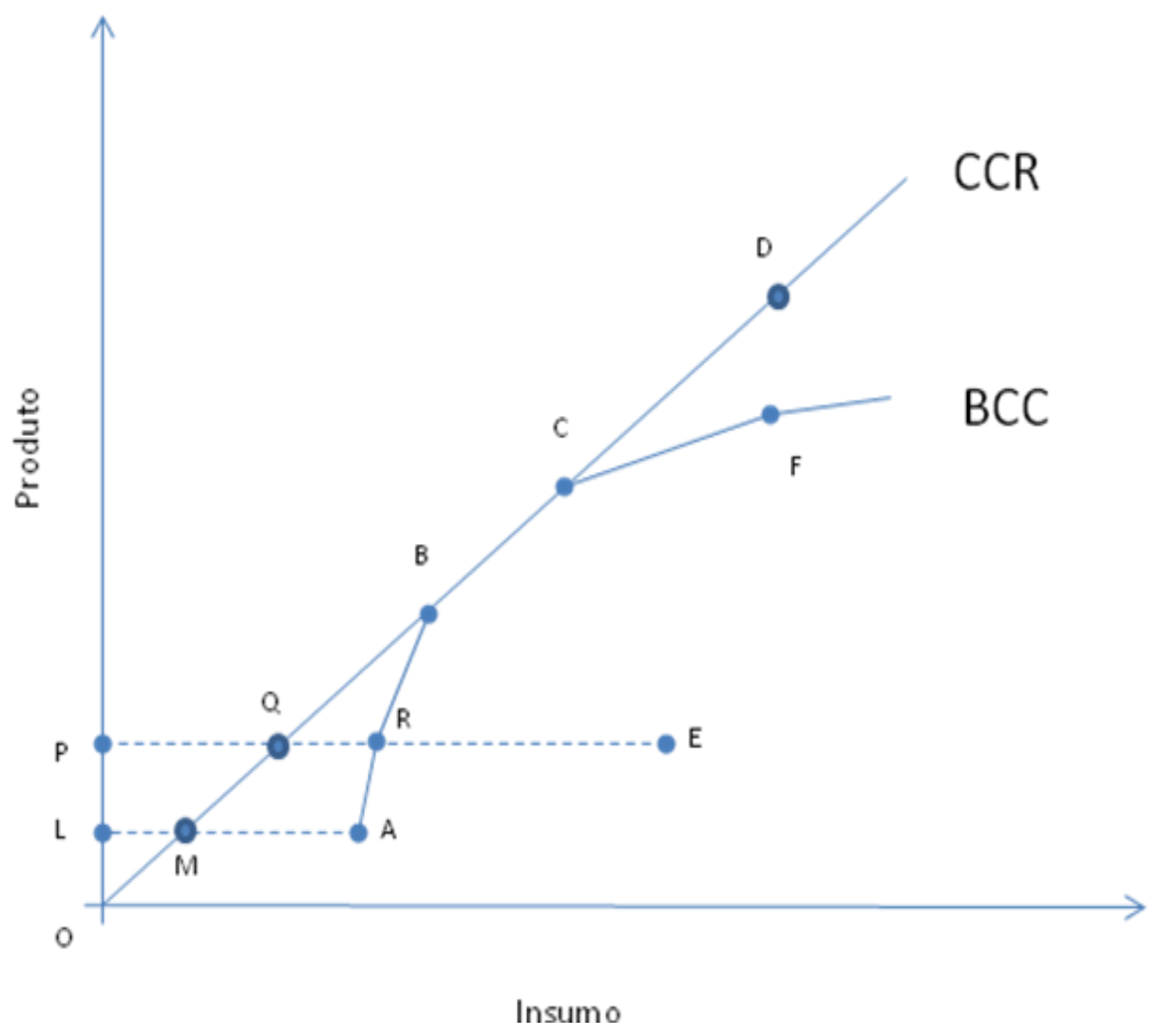

Fonte: Cooper, Seiford, Tone, 2006.

As UTD localizadas nos pontos M, Q, B, C e D são globalmente eficientes, segundo o modelo CCR. As UTD localizadas nos pontos A, R e $F$ são localmente eficientes, segundo o modelo BCC. O modelo BCC também considera as UTD dos pontos B e C como eficientes. A UTD do ponto $\mathrm{F}$, por exemplo, apesar de ser localmente eficiente, não apresenta uma eficiência global, pois seria possível reduzir muito o insumo sem ocorrer uma grande redução do produto. Por outro lado, seria possível elevar o escore de eficiência global da UTD do ponto A aumentando um 
pouco o insumo, já que haveria um grande acréscimo na quantidade de produto.

A UTD do ponto $E$ é ineficiente, tanto localmente como globalmente. 0 escore de eficiência de uma UTD varia de 0 a 1 . A UTD é eficiente se o seu escore é igual a 1 e ineficiente se for menor que 1.

A eficiência local da UTD em E é igual a: $E L=\frac{P R}{P E}$. A eficiência global dessa UTD é calculada como: $\mathrm{EG}=\frac{P Q}{P E}$. 
Há ainda uma eficiência devido à escala de produção. No caso da UTD localizada no ponto $\mathrm{E}$ a eficiência de escala é expressa como: $\mathrm{ES}=$ $\frac{P Q}{P R}$. A eficiência de escala (ES) é igual a: ES $=\frac{\text { eficiência global }}{\text { eficiência local }}=\frac{E G}{E L}=\frac{\frac{P Q}{P E}}{\frac{P R}{P E}}$ $=\frac{P Q}{P R}$. Assim, a eficiência de escala é calculada como o quociente da divisão do escore gerado pelo modelo CCR pelo escore do modelo BCC.

Portanto, a eficiência global (EG) corresponde à eficiência local (EL) multiplicada pela eficiência de escala (ES). Assim, EG $=\left(\right.$ EL) $x(E S)=\frac{P R}{P E}$ $\frac{P Q}{P R}=\frac{P Q}{P E}$.

Na figura 1, a UTD correspondente ao ponto A é localmente eficiente, mas globalmente ineficiente devido à ineficiência de escala.

Anteriormente, mostra-se a situação de eficiência de uma UTD no caso da utilização de um insumo e produção de um produto. Mas, em um sistema de produção de uma UTD há, geralmente, mais de um insumo e mais de um produto. Assim, segundo a descrição de Cooper, Seiford e Tone (2006), considera-se que os " $m$ " insumos e os "s" produtos da UTD ${ }_{\mathrm{j}}$ sejam, respectivamente, $\left(\mathrm{x}_{1 \mathrm{j}}, \mathrm{x}_{2 \mathrm{j}}, \ldots . . \mathrm{x}_{\mathrm{mj}}\right)$ e $\left(\mathrm{y}_{1 \mathrm{j}}, \mathrm{y}_{2 \mathrm{j}}\right.$, (..... $\left.y_{s j}\right)$.

Um dos modos de determinar a eficiência técnica da j-ésima UTD é por meio da maximização do quociente entre a soma dos valores ponderados dos produtos e a soma dos valores ponderados dos insumos. Segundo Cooper, Seiford e Tone (2006), considerando a soma dos valores ponderados dos insumos igual a 1, essa maximização é realizada por meio de programação linear, em relação a cada uma das "n" UTD $\mathrm{UT}_{\mathrm{j}}(\mathrm{j}=1,2, \ldots \mathrm{n})$. Por meio dessa programação, obtêm-se os valores para os pesos $v_{i}(i=1,2, \ldots . m)$ que ponderam os insumos e os valores para os pesos $u_{r}(r=1,2, \ldots . s)$ que ponderam os produtos em relação à $U T D_{j}$. Esses pesos são as variáveis do problema.

Segundo Cooper, Seiford e Tone (2006), considerando que a UTD。 seja a unidade que está sendo avaliada, ("o" varia de 1,2 , ...até n) o problema de programação transformado em uma forma linear é expresso como:

$\left(\mathrm{LP}_{0}\right) \max \theta=\mathrm{u}_{1} \mathrm{y}_{10}+\ldots \ldots . . . \mathrm{u}_{\mathrm{s}} \mathrm{y}_{\mathrm{so}}$ 
Sujeito a:

$$
\begin{aligned}
& v_{1} x_{10}+\ldots . .+v_{m} x_{m o}=1 \\
& u_{1} y_{1 j}+\ldots . .+u_{s} y_{s j} \leq v_{1} x_{1 j}+\ldots . .+v_{m} x_{m j} \quad(j=1,2, \ldots \ldots n) \\
& v_{1}, v_{2}, \ldots \ldots . v_{m} \geq 0 \\
& u_{1}, u_{2}, \ldots \ldots . . u_{s} \geq 0
\end{aligned}
$$

Neste problema de programação linear, maximizar

$$
\theta=\sum_{r=1}^{s} u_{r} y_{r o}
$$

é equivalente a maximizar o quociente entre a soma dos valores ponderados dos produtos e a soma dos valores ponderados dos insumos, ou seja, maximizar:

$$
\theta=\frac{\sum_{r=1}^{s} u_{r} y_{r o}}{\sum_{i=1}^{m} v_{i} x_{i o}}
$$

dado que o denominador, nesta última expressão, é igual a 1 .

Os valores de $(v, u)$ resultantes da programação indicam quais seriam as ponderações dos insumos e dos produtos mais favoráveis para a $U_{T D}$, no sentido de maximizar o quociente entre as somas dos valores ponderados. Em outras palavras, indicam a importância relativa de cada insumo e produto para a UTDo ser eficiente (COOPER, SEIFORD e TONE, 2006).

No problema de programação acima, os valores de $y_{j}$ variam da coluna 1 a "s" e da linha $j=1$ até $j=n$. Do mesmo modo, os valores $x_{j}$ variam da coluna $1 \mathrm{a} m$ e da linha $j=1 \mathrm{a} j=n$. Segundo Cooper, Seiford e Tone (2006), expressando essas variáveis na forma de matrizes $\mathrm{Y}$ e $\mathrm{X}$, o problema de programação acima pode ser expresso como:

$\left(L P_{0}\right)$ max uyo

sujeito a:

$$
\mathrm{vx}_{0}=1
$$




$$
\begin{gathered}
-v X+u Y \leq 0 \\
v \geq 0, u \geq 0
\end{gathered}
$$

Para a avaliação da eficiência técnica global, existem dois modelos CCR. Um modelo examina o produto máximo que pode ser obtido pela UTD com dada quantidade de insumos. Esse é o enfoque do modelo descrito acima. No software disponível em Cooper, Seiford e Tone (2006), o modelo é designado por CCR-O (output-oriented).

Outro enfoque é examinar a quantidade mínima de insumos necessária para a produção de certa quantidade de produto. No software acima referido, esse modelo é designado por CCR-I (inputoriented). 
Segundo Cooper, Seiford e Tone (2006), o modelo CCR-I é expresso como um modelo dual do $\mathrm{LP}_{0}$, ou seja:

$\left(D^{2} P_{0}\right) \min \theta$

Sujeito a;

$$
\begin{aligned}
& \theta \mathrm{X}_{0}-\mathrm{X} \lambda \geq 0 \\
& \mathrm{Y} \lambda \geq \mathrm{y}_{0} \\
& \lambda \geq 0
\end{aligned}
$$

Cooper, Seiford e Tone (2006) apresentam a correspondência entre as restrições e variáveis dos modelos $L P_{0}$ e $D L P_{0}$, conforme consta na tabela 1.

\begin{tabular}{|c|c|c|c|}
\hline $\begin{array}{l}\text { Restrições } \\
\text { Modelo LP }\end{array}$ & $\begin{array}{l}\text { Variáveis } \\
\text { Modelo DLP }\end{array}$ & $\begin{array}{l}\text { Restrições } \\
\text { Modelo DLP }\end{array}$ & $\begin{array}{l}\text { Variáveis } \\
\text { Modelo LP } 0\end{array}$ \\
\hline$v x_{0}=1$ & $\Theta$ & $\theta x_{0}-X \lambda \geq 0$ & $\mathbf{v} \geq \mathbf{0}$ \\
\hline$-v X+u Y \leq 0$ & $\lambda \geq 0$ & $\mathrm{Y} \lambda \geq \mathrm{y}_{0}$ & $\mathbf{u} \geq \mathbf{0}$ \\
\hline
\end{tabular}

Tabela 1. Correspondência entre variáveis e restrições dos modelos $L P_{0}$ e DLP 0

Fonte: Cooper, Seiford e Tone (2006).

Neste estudo, para analisar a eficiência técnica global das UTDs, utiliza-se o modelo CCR-I (input-oriented).

O modelo que considera retornos variáveis de escala, determinando a eficiência técnica local da UTD, por meio da minimização dos insumos para um dado nível de produto é denominado de BCC-I (input-oriented).

Segundo Cooper, Seiford e Tone (2006), esse modelo é expresso como:

$\operatorname{Min} \theta_{\mathrm{B}}$

Sujeito a:

$\theta_{\mathrm{B}} \mathrm{X}_{0}-\mathrm{X} \lambda \geq 0$

$\mathrm{Y} \lambda \geq \mathrm{y}_{0}$

$\mathrm{e} \lambda=1$

$\lambda \geq 0$ 
onde $\theta_{B}$ é um escalar.

Após serem obtidos os escores de eficiência técnica das lavouras de arroz, procura-se determinar, na próxima seção, as variáveis explicativas dos mesmos, por meio do modelo de regressão Tobit.

\subsection{Modelo de regressão Tobit}

Neste estudo, são considerados os escores de eficiência técnica global, ou seja, os valores obtidos por meio do modelo CCR-I. Esse modelo é mais restritivo na determinação da eficiência do que o BCC-I. Nem toda UTD localmente eficiente (modelo BCC-I) é globalmente eficiente (modelo CCR-I). Porém, toda UTD eficiente, segundo o modelo CCR-I, é também eficiente segundo o modelo BCC-I. Esses escores de eficiência constituem a variável explicada de uma regressão estatística. Nas UTD eficientes, o valor do escore atinge o máximo que é 1 . Portanto, a variável dependente é limitada a esse valor máximo.

Wooldridge (2003) observa que, neste caso, com a variável dependente limitada a um valor máximo (ou mínimo, ou ambos), o método de regressão que utiliza a técnica dos mínimos quadrados ordinários não é adequado. Esta situação é designada de amostra censurada. O modelo de regressão aconselhado é o Tobit.

A formulação geral do modelo Tobit, como apresentado por Gonçalves, Vieira, Lima e Gomes (2008), é:

$y_{i}^{*}=X_{i} \beta+\varepsilon_{i}$ onde $y_{i}^{*}$ é a variável dos escores; $X_{i}$ o vetor das variáveis explicativas; e $\beta$ o vetor dos parâmetros a serem estimados. Assume-se que os erros sejam normalmente distribuídos com média zero e variância $\sigma^{2}$.

Considerando o limite do escore igual a $1\left(\mathrm{y}^{\mathrm{c}}=1\right)$, as variáveis observadas $\left(\mathrm{y}_{\mathrm{i}}\right)$ são definidas como:

Se $y_{i}^{*}<\mathrm{y}^{\mathrm{c}}$, então $y_{i}=y_{i}^{*}$

Se $y_{i}^{*} \geq \mathrm{y}^{\mathrm{c}}$, então $y_{i}=y_{i}^{c}$

A estimativa dos parâmetros do modelo é geralmente realizada por meio do método de máxima verossimilhança. O software utilizado neste estudo para a estimativa dos parâmetros do vetor $\beta$ e o desvio padrão dos erros da regressão $\sigma$ é o E-Views. 
Para se avaliar o impacto marginal das variáveis explicativas contínuas sobre a variável dependente, é necessário multiplicar o valor do coeficiente $\beta_{\mathrm{j}}$ por um fator multiplicativo (Wooldridge, (2003).

Assim:

$$
E M_{X_{j}}=\frac{\partial E\left(y_{i}\right)}{\partial x_{j}}=\beta_{j} \Phi(z) \quad \mathrm{j}=1,2, \ldots, \mathrm{K} .
$$

onde $E \mathrm{M}_{\mathrm{j}}$ é o efeito marginal da variável independente contínua $\mathrm{x}_{\mathrm{j}}$ sobre a variável dependente $y_{i}$. O fator multiplicativo é $\Phi(z)$, que é o valor acumulado da distribuição normal padronizada z. O valor do z é: $z=\frac{x \beta}{\sigma}$ onde $\beta$ é o vetor dos parâmetros estimados e $\mathrm{x}$ são os valores médios das variáveis explicativas. $\mathrm{O}$ fator multiplicativo se situa entre 0 e 1 .

Segundo Wooldridge (2003), no modelo Tobit, o efeito marginal de uma variável explicativa binária $x_{1}$ seria obtido pela estimativa de $\mathrm{E}(\mathrm{y} \mid \mathrm{x})$ por meio da seguinte equação: $\mathrm{E}(\mathrm{y} \mid \mathrm{x})=\Phi(z) \times \beta+\sigma \phi(z)$, onde $\phi(z)$ é a função de densidade normal padronizada. Estima-se, inicialmente, $E(y \mid x)$ com $x_{1}=1$ e depois com $x_{1}=0$ e as outras variáveis seriam consideradas iguais aos seus valores médios. Para o autor, o valor de $\phi(z)$ é calculado por meio da função: $\phi(z)=(2 \pi)^{-1 / 2} \exp \left(-z^{2} / 2\right)$.

Com base nos métodos apresentados, na próxima seção são discutidos os principais resultados obtidos.

\section{RESULTADOS}

Neste estudo, são analisadas 8.693 lavouras de arroz irrigado do Rio Grande do Sul2. O censo do IRGA abrange as regiões produtoras de arroz irrigado do Rio Grande do Sul denominadas: Campanha, Depressão Central, Fronteira Oeste, Planície Costeira Externa à Lagoa dos Patos, Planície Costeira Interna à Lagoa dos Patos e Zona Sul (IRGA, 2006).

A Tabela 2 apresenta o número de lavouras consideradas localmente eficientes por região produtora e faixa de rendimento de

\footnotetext{
2 Não são consideradas as unidades (lavouras) que não possuem informação para todas as variáveis utilizadas.
} 
escala (modelo BCC). Na última coluna da tabela, apresenta-se o número total de lavouras consideradas na respectiva região.

Tabela 2. Número de lavouras localmente eficientes (pelo modelo BCC) por região produtora e faixa de rendimentos de escala

\begin{tabular}{|c|c|c|c|c|c|}
\hline \multirow[t]{2}{*}{ Região } & \multicolumn{3}{|c|}{$\begin{array}{l}\text { Número de lavouras por } \\
\text { rendimentos de escala }\end{array}$} & \multirow{2}{*}{$\begin{array}{l}\text { Total de } \\
\text { lavouras } \\
\text { eficiente } \\
\mathrm{s}\end{array}$} & \multirow{2}{*}{$\begin{array}{l}\text { Número de } \\
\text { lavouras da } \\
\text { região }\end{array}$} \\
\hline & $\begin{array}{c}\text { Constant } \\
\text { es }\end{array}$ & $\begin{array}{c}\text { Crescente } \\
\mathrm{s}\end{array}$ & $\begin{array}{c}\text { Decrescent } \\
\text { es }\end{array}$ & & \\
\hline Campanha & 24 & 34 & 2 & 60 & 1109 \\
\hline Depressão Central & 37 & 86 & 3 & 126 & 3274 \\
\hline Fronteira Oeste & 44 & 35 & 8 & 87 & 962 \\
\hline $\begin{array}{l}\text { Planície Costeira Externa à } \\
\text { Lagoa dos Patos }\end{array}$ & 32 & 94 & 2 & 128 & 1429 \\
\hline $\begin{array}{l}\text { Planície Costeira Interna à } \\
\text { Lagoa dos Patos }\end{array}$ & 12 & 49 & 4 & 65 & 1340 \\
\hline Zona Sul & 20 & 11 & 5 & 36 & 579 \\
\hline Total do RS & 169 & 309 & 24 & 502 & 8693 \\
\hline
\end{tabular}

Fonte: cálculo dos autores com base no Censo da lavoura orizícola 2005 do IRGA (2006).

Verifica-se que o maior número total de lavouras localmente eficientes situa-se na região da Planície Costeira Externa à Lagoa dos Patos (128 lavouras), seguida da Depressão Central (126 lavouras). As lavouras que são eficientes com rendimentos constantes de escala são eficientes, também, em termos globais. Do total de lavouras de arroz no Estado do RS, 169 são eficientes tecnicamente em termos globais. Já 309 lavouras são eficientes com rendimentos crescentes de escala (apenas localmente). Para tornarem-se eficientes em termos globais, essas lavouras devem aumentar a sua dimensão. As regiões que predominantemente apresentam essa característica são a Planície Costeira Externa e Interna de Lagoa dos Patos e Depressão Central.

Conforme a tabela 2, as lavouras eficientes com rendimentos decrescentes de escala são $24 \mathrm{em}$ todo o estado. Para atingirem a eficiência em termos globais, neste caso seria necessário reduzir a sua dimensão. Embora o número de lavouras com essa característica seja pequeno, as regiões com maior presença em número de lavouras é a Fronteira Oeste e a Zona Sul do estado. 
A tabela 3 apresenta a quantidade de lavouras eficientes em relação ao total em termos percentuais para as regiões do estado. A Fronteira Oeste, seguida da Planície Costeira Externa à Lagoa dos Patos são as duas regiões que apresentam o maior percentual de lavouras localmente eficientes $(9,04 \%$ e $8,96 \%$, respectivamente). A região com o menor percentual de lavouras eficientes $(3,85 \%)$ é a Depressão Central. As regiões Fronteira Oeste, Planície Costeira Externa à Lagoa dos Patos e Zona Sul apresentam lavouras eficientes acima do percentual médio do estado, que é $5,77 \%$. As demais regiões apresentam percentuais inferiores de lavouras eficientes em comparação à média do estado.

Tabela 3. Percentual de lavouras localmente eficientes (pelo modelo $\mathrm{BCC}$ ) por faixa de rendimentos de escala em cada região produtora

\begin{tabular}{|c|c|c|c|c|}
\hline \multirow[t]{2}{*}{ Região } & \multicolumn{3}{|c|}{$\begin{array}{l}\text { Percentual de lavouras por } \\
\text { rendimentos de escala }\end{array}$} & \multirow{2}{*}{$\begin{array}{l}\text { Percentual total } \\
\text { de lavouras } \\
\text { eficientes }\end{array}$} \\
\hline & Constantes & $\begin{array}{l}\text { Crescente } \\
\text { s }\end{array}$ & $\begin{array}{l}\text { Decrescent } \\
\text { es }\end{array}$ & \\
\hline Campanha & 2,16 & 3,07 & 0,18 & 5,41 \\
\hline Depressão Central & 1,13 & 2,63 & 0,09 & 3,85 \\
\hline Fronteira Oeste & 4,57 & 3,64 & 0,83 & 9,04 \\
\hline $\begin{array}{l}\text { Planície Costeira Externa } \\
\text { à Lagoa dos Patos }\end{array}$ & 2,24 & 6,58 & 0,14 & 8,96 \\
\hline $\begin{array}{l}\text { Planície Costeira Interna } \\
\text { à Lagoa dos Patos }\end{array}$ & 0,90 & 3,66 & 0,30 & 4,85 \\
\hline Zona Sul & 3,45 & 1,90 & 0,86 & 6,22 \\
\hline Total do RS & 1,94 & 3,55 & 0,28 & 5,77 \\
\hline
\end{tabular}

Fonte: cálculo dos autores com base na Tabela 2 .

A tabela 3 mostra que apenas 5,77\% (502 lavouras) são eficientes localmente no RS. Em termos percentuais, a região da Fronteira Oeste (9,04\%), Planície Costeira Externa à Lagoa dos Patos (8,96\%) e Zona Sul $(6,22 \%)$ apresentam as maiores proporções de lavouras eficientes em comparação à média do RS.

Um menor percentual das lavouras localmente eficientes são globalmente eficientes, operando na faixa de rendimentos constantes de escala. Para o RS apenas $1,94 \%$ do total do RS (169 lavouras) são globalmente eficientes. Destaca-se a região da Fronteira Oeste $(4,57 \%)$ e da Zona Sul $(3,45 \%)$ por apresentarem os maiores percentuais de eficiência global no RS. 
Para as lavouras que estão na faixa dos rendimentos crescentes de escala no RS (3,55\%), existe a necessidade de aumentar a escala de produção para serem eficientes em termos globais. Isso pode ser difícil ou inviável pela pouca disponibilidade de terra e de recursos financeiros.

Um pequeno número de lavouras localmente eficientes na faixa dos rendimentos decrescentes de escala no estado $(0,28 \%)$ pode reduzir a escala para se tornarem globalmente eficientes. Porém, nesse caso, para reduzir a escala seria necessário que houvesse uma segunda opção para a área de terra, equipamentos e mão de obra que seriam eliminados do cultivo do arroz. Essa é uma limitação da análise aqui realizada, pois inexistem informações sobre as alternativas de produção disponíveis em outras atividades agropecuárias junto às lavouras de arroz.

De uma forma geral, para o estado do RS, as lavouras de arroz apresentam um elevado percentual de ineficiência em termos locais, mostrando que existe a possibilidade de aumentar a eficiência nas lavouras de arroz a partir do melhor uso dos recursos disponíveis para a produção de arroz. O próximo passo seria por meio da busca de uma eficiência em termos globais. Os resultados mostram que a maior parte das lavouras do RS precisa aumentar a escala de produção para atingir esse segundo patamar de eficiência, a global.

Conforme descrito anteriormente, foi realizada uma análise de regressão estatística de variáveis explicativas sobre o índice de eficiência técnica global das lavouras, ou seja, os valores obtidos por meio do modelo CCR-I. O quadro 1 descreve as variáveis independentes ou explicativas utilizadas na regressão estatística.

Quadro 1. Variáveis explicativas consideradas na regressão estatística

\begin{tabular}{|l|l|}
\hline \multicolumn{1}{|c|}{ Código } & \multicolumn{1}{c|}{ Descrição } \\
\hline Escosecun & $\begin{array}{l}\text { Variável binária (1 ou 0), igual a } 1 \text { se o produtor tem } \\
\text { escolaridade secundária. }\end{array}$ \\
\hline Escouniv & $\begin{array}{l}\text { Variável binária, igual a } 1 \text { se o produtor tem escolaridade } \\
\text { universitária ou pós-graduação. }\end{array}$ \\
\hline Pterraprop & Percentual da área de lavoura que é terra própria. \\
\hline Paguaprop & Percentual da área de lavoura irrigada com água própria. \\
\hline Pareairrignat & Percentual da área de lavoura com irrigação natural. \\
\hline asstecnicasim & Variável binária, igual a 1 se o produtor utiliza assistência \\
\hline
\end{tabular}




\begin{tabular}{|c|c|}
\hline & técnica. \\
\hline Semoutu & Percentual da área de lavoura semeada em outubro. \\
\hline Semnove & Percentual da área de lavoura semeada em novembro. \\
\hline Cultmini & Percentual da área semeada no sistema de cultivo mínimo. \\
\hline Plandireto & Percentual da área semeada no sistema de plantio direto. \\
\hline Pregermi & Percentual da área semeada no sistema pré-germinado. \\
\hline Regzs & Variável binária. Região da lavoura é a Zona Sul \\
\hline Regcelp & $\begin{array}{l}\text { Variável binária. Região da lavoura é a Planície Costeira } \\
\text { Externa à Lagoa dos Patos. }\end{array}$ \\
\hline Regcilp & $\begin{array}{l}\text { Variável binária. Região da lavoura é a Planície Costeira } \\
\text { Interna à Lagoa dos Patos. }\end{array}$ \\
\hline Regcamp & Variável binária. Região da lavoura é a Campanha. \\
\hline Regfroe & Variável binária. Região da lavoura é a Fronteira Oeste. \\
\hline
\end{tabular}

A regressão foi realizada por meio do modelo Tobit, utilizando o software EViews. Os resultados da regressão dos índices de eficiência técnica global (gerados pelo modelo $\mathrm{CCR}-\mathrm{I}$ ) das lavouras sobre as variáveis explicativas, descritas no quadro 1 , são apresentados na tabela 4. 
Tabela 4- Resultados da regressão dos índices de eficiência técnica sobre as variáveis explicativas

\begin{tabular}{l|c|c|c|c}
\hline $\begin{array}{l}\text { Variáveis } \\
\text { Explicativas }\end{array}$ & Coeficiente & Estatística Z & Probabilidade & $\begin{array}{c}\text { Efeito } \\
\text { marginal }\end{array}$ \\
\hline Constante & 0,4843 & 49,58 & 0,00 & \\
\hline Escosecun & 0,0091 & 1,82 & 0,07 & 0,0091 \\
\hline Escouniv & 0,0286 & 4,27 & 0,00 & 0,0285 \\
\hline Pterraprop & $-0,0001$ & $-2,88$ & 0,00 & $-0,0001$ \\
\hline Paguaprop & $-0,0001$ & $-2,40$ & 0,02 & $-0,0001$ \\
\hline Pareairrignat & $-0,0007$ & $-15,76$ & 0,00 & $-0,0007$ \\
\hline asstecnicasim & 0,0309 & 6,61 & 0,00 & 0,0307 \\
\hline Semoutu & 0,0009 & 9,58 & 0,00 & 0,0009 \\
\hline Semnove & 0,0005 & 5,24 & 0,00 & 0,0005 \\
\hline Cultmini & 0,0005 & 9,25 & 0,00 & 0,0005 \\
\hline Plandireto & 0,0006 & 4,78 & 0,00 & 0,0006 \\
\hline Pregermi & 0,0004 & 6,76 & 0,00 & 0,0004 \\
\hline Regzs & 0,0457 & 5,23 & 0,00 & 0,0454 \\
\hline Regcelp & 0,0361 & 5,89 & 0,00 & 0,0359 \\
\hline Regcilp & $-0,0029$ & $-0,44$ & 0,66 & 0,0000 \\
\hline Regcamp & 0,0549 & 8,01 & 0,00 & 0,0545 \\
\hline Regfroe & 0,0644 & 8,72 & 0,00 & 0,0640 \\
\hline Escala & 0,1833 & 129,77 & 0,00 & \\
\hline R2 ajustado & 0,12 & & & \\
\hline Fonte: cálculos & $a, 005 \mathrm{nas}$ & & \\
\hline
\end{tabular}

Fonte: cálculos dos autores baseados no censo da lavoura orizícola 2005 (IRGA, 2006).

A estimativa do efeito marginal das variáveis explicativas contínuas sobre as variáveis dependentes tem um valor calculado de $\mathrm{z}$ igual a 2,9013. O valor acumulado da distribuição normal padronizada até esse valor de z, o fator multiplicativo $\Phi(z)$, é igual a 0,9981. Assim, o efeito marginal de cada variável explicativa contínua sobre a variável dependente é, praticamente, igual ao valor do próprio coeficiente $\beta_{\mathrm{j}}$. No caso das variáveis binárias, os efeitos marginais também são semelhantes aos valores dos coeficientes $\beta_{\mathrm{j}}$. Esses efeitos marginais estão apresentados na tabela 4 , acima.

Com base nos resultados da tabela 4 , as cinco regiões incluídas têm como referência a região da Depressão Central. Quando comparadas às diferenças entre as regiões de produção, pode-se concluir que as lavouras da Fronteira Oeste tendem a ser mais eficientes do que as demais lavouras do estado. O índice de eficiência dessas lavouras tende a ser 0,0640 maior do que o das lavouras da Depressão 
Central e da Planície Costeira Interna à Lagoa dos Patos (onde o efeito marginal é igual a zero).

Em uma situação intermediária, estão as regiões da Campanha, da Zona Sul e da Planície Costeira Externa à Lagoa dos Patos, que apresentam uma média superior nos índices de eficiência de 0,0545, 0,0454 e 0,0359, respectivamente.

Os maiores índices de eficiência apresentados por uma região podem ser devido a fatores não incluídos nesta análise, como, por exemplo, as diferenças em termos de condições edafoclimáticas. Portanto, as diferenças observadas não podem ser atribuídas a priori exclusivamente pela maior eficiência do produtor local. Cabe, porém, uma citação da publicação do censo da lavoura de arroz (IRGA, 2006) que afirma que a Fronteira Oeste apresenta a maior concentração de universitários, com 238 entrevistados. Como mencionado acima, essa é a região com o maior índice de eficiência.

Observa-se que o índice de eficiência da lavoura aumenta 0,0091 quando o produtor possui ensino médio completo e se eleva 0,0285 quando a sua escolaridade é universitária ou de pós-graduação. As diferenças em termos de capital humano mostraram-se relevantes para explicar o melhor desempenho em termos de eficiência técnica nas lavouras de arroz do estado. Quanto maior o nível educacional do produtor maior a eficiência técnica nas lavouras de arroz.

Um aumento de $1 \%$ no percentual da área de lavoura com terra própria reduz o índice de eficiência em 0,0001 . A elevação em $1 \%$ da área irrigada com água própria e com irrigação natural reduz o índice de eficiência em 0,0001 e 0,0007, respectivamente. Isso pode ser explicado pelo fato de que, quem paga pelos recursos, terra e água arrendada e combustível para fazer a irrigação, utiliza os fatores de produção de modo mais eficiente. Esse resultado está de acordo com as conclusões de Helfand e Levine (2004). Segundo esses autores, os arrendatários da terra que pagam valor pré-fixado pelo arrendamento são $14 \%$ mais eficientes do que os proprietários.

A utilização da assistência técnica pelos produtores aumenta o índice de eficiência técnica das lavouras em 0,0307. Isso mostra que, juntamente com o nível educacional e regiões de cultivo, a assistência técnica é importante para alcançar níveis mais elevados de eficiência técnica na produção de arroz no Estado do RS. 
Para aumentos de $1 \%$ na área semeada nos meses de outubro e novembro (período recomendado para plantio), aumenta o índice de eficiência técnica da lavoura em 0,0009 e 0,0005, respectivamente. Os sistemas de cultivo mínimo, plantio direto e pré-germinado conduzem a maiores índices de eficiência do que o plantio convencional. Para cada $1 \%$ de aumento na área semeada com cultivo mínimo, plantio direto ou pré-germinado, o índice de eficiência técnica aumenta em 0,0005, 0,0006 e 0,0004 , respectivamente.

Apesar da maioria dos produtores incluídos na análise apresentarem índices de ineficiência técnica nas lavouras de arroz do RS, os resultados obtidos indicam as principais variáveis que podem incrementar a eficiência. As variáveis que melhoram o índice de eficiência dos produtores de arroz no estado são: assistência técnica, nível de escolaridade e sistema de cultivo. Os resultados mostram que existem diferenças importantes entre as lavouras de arroz conforme a região do estado e a condição do produtor (proprietário ou arrendatário).

\section{CONCLUSÕES}

O estudo sobre eficiência técnica nas lavouras de arroz analisou um total de 8.693 lavouras de arroz no RS e verificou que apenas 5,7\% são eficientes (502 lavouras de arroz). O estudo mostra que existem diferenças importantes entre as regiões do estado. Aquelas que apresentaram maior percentual de lavouras de arroz eficiente estão na Fronteira Oeste e na Planície Costeira Externa à Lagoa dos Patos.

Os resultados do estudo sinalizam aos formuladores de política que haja um estímulo à realocação dos recursos escassos para vencer os obstáculos e incrementar a eficiência técnica. Entre os principais aspectos, destaca-se a necessidade de melhorias nos serviços de educação e na extensão rural. Nesse sentido, a identificação das fontes de ineficiência e dos usos mais eficientes da tecnologia disponível é fundamental para o desenvolvimento de políticas públicas e iniciativas privadas destinadas a melhorar as condições de vida dos produtores agrícolas, principalmente dos pequenos.

Por fim, cabe destacar a importância de existir uma política agrícola diferenciada conforme a localização espacial e o nível de 
eficiência regional das lavouras de arroz, fundamental para garantir uma melhor eficiência técnica. A exemplo disso destaca-se o efeito das diferenças entre as regiões em termos edafoclimáticos, sistema de plantio predominante e condição do produtor sobre o nível de eficiência técnica.

\section{REFERÊNCIAS}

ARNADE, C. Using a Programming Approach to Measure International Agricultural Efficiency and Productivity. Journal of Agricultural Economics, v. 49, n. 1, 1998.

CHAVAS, J. P. On the Economics of Agricultural Production. The Australian Journal of Agricultural and Resource Economics, v. 52, 2008.

CHAVAS, J. P; CHAMBERS, R. G; POPE, R. D. Production Economics and Farm Management: a Century of Contributions. American Journal of Agricultural Economics, v. 92 (2), 2010.

CHAVAS, J. P; PETRIE, R; ROTH, M. Farm Household Production Efficiency: Evidence from the Gambia. American Journal of Agricultural Economics, v.87(1), 2005.

CHARNES, A; COOPER, W. W; RHODES, E. Measuring the Efficiency of Decision Making Units. European Journal of Operational Research, v. 2, 1978.

COELLI, T; RAHMAN, S; THIRTLE, C. Technical, Allocative, Cost and Scale Efficiencies in Bangladesh Rice Cultivation: a Non-Parametric Approach. Journal of Agricultural Economics, v. 53, n. 3, 2002.

COOPER, W. W; SEIFORD, L. M; TONE, K. Introduction to Data Envelopment Analysis and its Uses. Springer Science + Business Media, Inc. New York, 2006.

DHUNGANA, B. R; NUTHALL, P. L; NARTEA, G. V. Measuring the Economic Inefficiency of Nepalese Rice Farms Using Data Envelopment 
Analysis. The Australian Journal of Agricultural and Resource Economics, v. 48, n. 2, 2004.

FERREIRA FILHO, J. B. S; PEREIRA FILHO, C. A. Sources of Small Family Farm Production Inefficiency, Recôncavo Region, Bahia, Brazil. Revista de Economia e Sociologia Rural, v. 41, n. 1. Brasília, 2003.

GONÇALVES, R. M. L; VIEIRA, W. da C; LIMA, J. E; GOMES, S. T. Analysis of Technical Efficiency of Milk-Producing Farms in Minas Gerais. Economia Aplicada, v. 12, n. 2. São Paulo, 2008.

HELFAND, S. M; LEVINE, E. S. Farm Size and the Determinants of Productive Efficiency in the Brazilian Center West. Agricultural Economics, v. 31, 2004.

IRGA (Instituto Riograndense do Arroz). Censo da Lavoura de Arroz Irrigado do Rio Grande do Sul - Safra 2004/05. Porto Alegre: IRGA Política Setorial, 2006.

VICENTE, J. R. Economic Efficiency of Agricultural Production in Brazil. Revista de Economia e Sociologia Rural, v.42, n.2. Brasília, 2004.

WOOLDRIDGE, J. M. Introductory Econometrics-A Modern Approach. Thomson South-Western, 2003.

Submetido em 28/11/2012

Aprovado em $08 / 12 / 2014$

Sobre os autores

Augusto Mussi Alvim

Eng. Agrônomo, Doutor em Economia. Professor do Departamento de Ciências Econômicas e do Programa de Pós-Graduação em Economia - PPGE da Pontifícia Universidade Católica do Rio Grande do Sul (PUCRS). Pesquisador do CNPq.

Endereço: Av. Ipiranga, 6681 - prédio 50, Partenon. 90619900 - Porto Alegre - RS Brasil.

E-mail: augusto.alvim@pucrs.br

Valter José Stülp

Redes (St. Cruz Sul, Online), v. 20, n² 2, p. 158 - 175, maio/ago. 2015182 
Eng. Agrônomo, PhD em Economia Agrícola. Professor do Departamento de Ciências Econômicas e do Programa de Pós-Graduação em Economia - PPGE da Pontifícia Universidade Católica do Rio Grande do Sul (PUCRS)

Endereço: Av. Ipiranga, 6681 - prédio 50, Partenon. 90619900 - Porto Alegre - RS Brasil.

E-mail: stulp@terra.com.br

\section{Victor Hugo Kayser}

Eng. Agrônomo, Mestre em Economia Rural. Pesquisador do Instituto Riograndense do Arroz - IRGA.

E-mail: victor-kayser@irga.rs.gov.br 